\begin{tabular}{l}
\hline OPEN OACCESS Freely available online \\
http://www.banglajol.info/index.php/BJID/index \\
Original Article \\
Bangladesh Journal of Infectious Diseases \\
June 2016, Volume 3, Number 1 \\
ISSN (Online) 2411-670X \\
ISSN (Print) 2411-4820
\end{tabular}

\title{
Comparison of the Lytic Blood Culture Method with the Conventional Blood Culture Method in Cases of Enteric Fever in a Tertiary Care Hospital
}

\author{
Safia Sultana ${ }^{1}$, Md. Akram Hossain ${ }^{2}$, Md. Abdoullah Al Maruf ${ }^{3}$, Mohammad Abdul Gani $^{4}$ \\ ${ }^{1}$ Assistant Professor, Department of Microbiology, Shaheed Syed Nazrul Islam Medical College, Kishoreganj, Bangladesh; \\ ${ }^{2}$ Professor of Microbiology, National Institute of Preventive and Social Medicine, Dhaka, Bangladesh; ${ }^{3}$ Residential Medical Officer, \\ 250 bed district hospital, Kishoreganj, Bangladesh; ${ }^{4}$ Assistant Professor, Department of Pharmacology, Shaheed Syed Nazrul Islam \\ Medical College, Kishoreganj, Bangladesh
}

[Received: 1 January 2015; Accepted: 15 July2015; Published: 1 June 2016]

\begin{abstract}
Background: Blood Culture is the gold standard and accurate method of diagnosing bacteremia in enteric fever; however, conventional blood culture is slow in isolating Salmonella typhi and Salmonella paratyphi. Objective: The main aim of this study was to compare the result of the lytic centrifugation method with conventional blood culture system for the accurate diagnosis of enteric fever in febrile patients. Methodology: The cross-sectional study was carried out in the department of Microbiology, Mymensingh Medical College, Mymensingh between July 2010 and June 2011 including 200 individuals of different ages and sexes. Of them, 150 were clinically suspected cases of typhoid fever and 50 controls comprising of 25 non-typhoidal febrile patients and 25 healthy individuals. Both types of blood culture were performed for each of the cases and controls. Result: The lytic centrifugation method recovered more organisms $(17.3 \%)$ than the conventional blood culture method (13.3\%). Time required for isolation of $S$. typhi and S. paratyphi A was short in lytic method (18-20hours) than conventional method (4272hours). Total contamination rate was $0.5 \%$ by lytic as compared to the conventional blood culture method which was $5.0 \%$. Conclusion: In conclusion the lytic method is better than conventional blood culture system for good result, short isolation time and less chance of contamination. [Bangladesh Journal of Infectious Diseases 2016;3(1):6-10]
\end{abstract}

Keywords: Enteric fever; blood culture; lytic method; saponin

[How to Cite this article: Sultana S, Hossain MA, Maruf MAA, Gani MA. Comparison of the Lytic Blood Culture Method with the Conventional Blood Culture Method in Cases of Enteric Fever in a Tertiary Care Hospital. Bangladesh J Infect Dis 2016;3(1):6-10]

Corresponding author: Dr. Safia Sultana, Assistant Professor, Department of Microbiology, Shaheed Syed Nazrul Islam Medical College, Kishoreganj, Bangladesh; Email: drsafia79@yahoo.com; Cell no: +8801712558404

Conflict of Interest: Authors have declared no conflict of interest.

Contributions to authors: MAA \& GMA have contributed from protocol preparation to manuscript writing as well as in data collection. HMA has guided, prepared and has revised the manuscript.

\section{Introduction}

Enteric fever is a systemic prolonged febrile illness caused by Salmonella typhi and Salmonella paratyphi $A, B, C$ are widely recognized as a major public health problem in many developing countries ${ }^{1}$. It is endemic in the Indian subcontinent including Bangladesh, South-East and Far- East Asia, the middle East, Africa, central and South America ${ }^{1}$. It is transmitted through the faecal oral 
route by the consumption of contaminated water and food, particularly raw or undercooked meat, poultry, eggs and milk. The consumption may occur either directly from person-to-person or by ingestion of food or water contaminated with faeces or urine carrier as well as through flies ${ }^{2}$. The disease may occur in all ages, with the highest incidence found particularly in children ${ }^{3}$. In addition infection is most common in young children and elderly with peak incidence in summer and fall ${ }^{4}$. It is therefore, presumed that enteric fever is a major health problem in all those parts of the world where safe drinking water and sanitation is inadequate. There was estimation that typhoid fever caused more than 21 million illnesses and more than 216,000 deaths in the world during $2000^{5}$. The incidence of cases of bacteraemic typhoid fever in Bangladesh per year was found 390/100,000 in $2001^{6}$. Enteric fever occurs only in the humans, which may turn into a severe infection progressing to complications and death. Persons with typhoid fever carry the causative agent $S$. typhi in their bloodstream and intestinal tract. S. typhi is uniquely adapt to humans and carriers represent the sole source of these organisms for a short period of time (convalescent carrier) or chronic carriers who shed the organism for longer than 1 year $^{7}$.

Since all the signs and symptoms of enteric fever are nonspecific, a definitive diagnosis of the disease depending on the clinical presentation alone is very difficult. Therefore, laboratory-based investigations are essential for supporting the diagnosis of the fever. Several different techniques are used for the diagnosis of the disease. The gold standard for diagnosis of enteric fever is the isolation of S. typhi and $S$. paratyphi from appropriate samples including blood, bone marrow aspirates, stool, urine and rose $\operatorname{spots}^{8-9}$. A lytic centrifugation technique of blood culture has some added advantage over conventional method to reduce the isolation time ${ }^{10}$.

There are some international studies on the lytic centrifugation method that showed it to be a better method than the conventional one ${ }^{11-14}$. The benefit of this system include; the more rapid and greater recovery of the organism; the presence of actual colonies for direct identification and susceptibility testing after initial incubation; the ability to quantify the colony forming units present in the blood; rapid detection of polymicrobial bacteraemia; and possible recovery of intracellular microorganisms caused by lysis of host cells. Limitation of the method is high rate of possible contamination $^{10-13}$.
In the perspective of Bangladesh, it is presumed that the diagnosis of enteric fever is usually based on clinical presentation as well as Widal test, both of which are associated with numerous limitations. The diagnosis of enteric fever on clinical presentations alone is difficult, as the presenting symptoms are diverse and similar to those observed with other febrile illnesses, especially during the first weeks of the infection ${ }^{1}$. The present study was designed to identify the cases of enteric fever employing the different techniques of blood culture keeping in mind the commonly occurring problems and to overcome them. For this reason, blood culture was done by lytic centrifugation method as well as conventional blood culture method to compare result and the length of isolation of S. typhi and $S$. paratyphi $A$ for the accurate diagnosis of enteric fever in febrile patients.

\section{Methodology}

This cross-sectional study was carried out in the Department of Microbiology, Mymensingh Medical College (MMC), Mymensingh, Bangladesh, from July, 2010 to June, 2011 for a period of one year. Patients of both sexes, representing all age groups were included who attended out patient departments (OPD) of Medicine and Paediatrics units of MMC Hospital, irrespective of their prior antibiotic, simultaneously for lytic centrifugation and conventional blood culture methods. Blood samples (taking one from each of the cases and controls) were analyzed using two blood culture methods concurrently for recovery of bacteria from suspected cases of enteric fever. Venous blood was collected aseptically following universal safety precaution. Total amount of whole blood was collected for lytic centrifugation as well as for conventional blood culture method was $2 \mathrm{ml}$ for children $0-2$ years, $4 \mathrm{ml}$ for $>2$ to 12 years and 10 $\mathrm{ml}$ for adults $>12$ years of age. The conventional blood culture method was using trypticase soy broth and the lytic centrifugation method was using saponin by centrifuging at $3000 \mathrm{~g}$ for 30 minutes. By the conventional method, blood was inoculated in blood culture bottles containing trypticase soy broth (1:5 dilutions). These were incubated at $35^{\circ} \mathrm{C}$ for 7 days and periodically shaken. The first subculture was done from each bottle within 12 to 24 hours on Blood agar and MacConkey's agar plates and then on fourth and seventh day. By lytic centrifugation method, the aseptically collected blood samples were collected in heparinized tube containing lytic solution which was made in our laboratory for initial processing. Blood sample were mixed well by inverting the tube 3-5 times to mix blood with lytic solution and mixture were shaked, 
vortexed and centrifuged at $3000 \mathrm{rpm}$ for 30 minutes. After centrifugation, the supernatant were discarded and $1 \mathrm{ml}$ of the deposit containing the pathogen is vigorously vortexed and entire sediment were directly inoculated onto blood agar and MacConkey's agar media. The inoculated culture plates were immediately placed in an incubator at $37^{\circ} \mathrm{C}$ for 24 to 48 hours. If there was growth by either the conventional or lytic centrifugation method, colonies of $S$. typhi and $S$. paratyphi $A$ were further identified by standard laboratory techniques ${ }^{15-16}$.

\section{Results}

Among 150 suspected cases of enteric fever included in this study, $17.3 \%$ isolates were recovered by the lytic centrifugation method and $13.3 \%$ by conventional method. The ability of each system to recover the salmonella species observed in this study is shown in (Table 1).

Table 1: Number of Isolates by Lytic Centrifugation and Conventional Blood Culture Methods

\begin{tabular}{|c|c|c|c|}
\hline $\begin{array}{l}\text { Bacteria } \\
\text { Isolated }\end{array}$ & 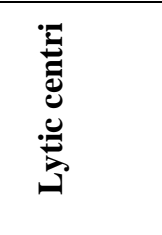 & 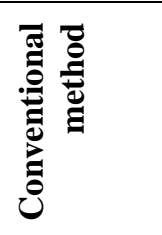 & 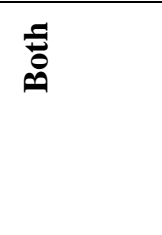 \\
\hline S. typhi & $23(15.3 \%)$ & $19(12.6 \%)$ & $42(28 \%)$ \\
\hline S. paratyphi A & $3(2 \%)$ & $1(0.66 \%)$ & $4(2.6 \%)$ \\
\hline Total & $26(17.3 \%)$ & $20(13.3 \%)$ & $46(30.6 \%)$ \\
\hline
\end{tabular}

Among 150 blood samples from the suspected cases, by lytic method 23(15.3\%) were culture positive for $S$. typhi and 3 (2\%) cases positive for $S$. paratyphi $A$.
By conventional method, 19(12.6\%) were culture positive for $S$. typhi and $1(0.66 \%)$ cases positive for $S$. paratyphi $A$.

Table 2: Time Required For Growth of Organisms by both Lytic Centrifugation and Conventional Blood Culture Method (Hours)

\begin{tabular}{lcc}
\hline $\begin{array}{l}\text { Bacteria } \\
\text { Isolated }\end{array}$ & $\begin{array}{l}\text { Lytic } \\
\text { centrifugation }\end{array}$ & $\begin{array}{l}\text { Conventional } \\
\text { methods }\end{array}$ \\
\hline S. typhi & 18 & 42 \\
S. paratyphi A & $16-24$ & 72 \\
\hline
\end{tabular}

Whereas, all of the 50 samples of the controls including non-typhoidal febrile patients and healthy persons were negative by both type of blood culture.

Table 3: Relationship of Blood Culture Positivity with Duration of Fever of the Cases

\begin{tabular}{|c|c|c|c|c|}
\hline \multirow{2}{*}{$\begin{array}{l}\text { Result of } \\
\text { blood } \\
\text { culture }\end{array}$} & \multicolumn{4}{|c|}{$\begin{array}{l}\text { No. }(\%) \text { of patients and duration of } \\
\text { illness in days }\end{array}$} \\
\hline & $1-3$ & $4-7$ & $8-10$ & $11-14$ \\
\hline Positive & $1(0.7)^{*}$ & $25(16.7)$ & $0(0.0 \%)$ & $0(0.0)$ \\
\hline Negative & $01(0.7)$ & $96(64.0)$ & $26(17.3)$ & $1(0.7)$ \\
\hline
\end{tabular}

The lytic centrifugation method recovered more organisms $(17.3 \%)$ than the conventional blood culture method (13.3\%). Time required for the growth of S. typhi and S. paratyphi A by both lytic centrifugation and conventional blood culture method is shown in (Table 2). S. typhi grew within 18 hours approximately by the lytic centrifugation method as compared to 42 hours (approx.) by the conventional method. S. paratyphi A grew within 16 to $24 \mathrm{~h}$ by lytic centrifugation method as compared to $72 \mathrm{~h}$ by the conventional method.

Table 4: Antibiotic Usage by the Blood Culture-Negative Suspected Cases of Enteric Fever

\begin{tabular}{llcc}
\hline Antibiotic & Dosage Schedule & Duration of Intake & No. $(\boldsymbol{\%})$ of cases \\
\hline Ciprofloxacin & $100-500 \mathrm{mg}$ twice daily & 2 to 7 days & $23(25.9 \%)$ \\
Cotrimoxazole & $960 \mathrm{mg}$ twice daily & 2 to 5 days & $14(22.2 \%)$ \\
Tetracycline & $250 \mathrm{mg}$ twice daily & 2 to 7 days & $9(5.6 \%)$ \\
Azithromycin & $500 \mathrm{mg}$ single & 3 days & $18(14.8 \%)$ \\
Amoxycillin & $250-500 \mathrm{mg}$ thrice daily & 3 to 5 days & $13(13.0 \%)$ \\
Levofloxacin & $500 \mathrm{mg}$ single & 3 to 7 days & $17(5.6 \%)$ \\
Cephradin & $250 \mathrm{mg}$ thrice daily & 6 days & $16(1.9 \%)$. \\
\hline
\end{tabular}

*14 patients taken some drugs but cannot mentioned the name properly 
Time to growth by lytic centrifugation was highly significant $(\mathrm{p}=0.0001)$, as compared to time to growth by the conventional blood culture method. By observing the relationship of blood culture positivity and duration of illness, it was found that all of the samples yielding positive result were collected from the cases complaining of 3-7 days of fever (Table 3). Considering the relationship of antibiotic usage with the results of blood culture of the suspected cases of enteric fever, it was found that most of the blood culture positive cases taken no antibiotic during the episode and majority of the blood culture negative cases gave history of taking different antibiotics for variable periods (Table 4). Total contamination by lytic centrifugation method was low $(0.5 \%)$, as compared to the conventional blood culture method (5.0\%).

\section{Discussion}

Overall bacteria recovered from 150 suspected cases of enteric fever were $17.3 \%$ by lytic centrifugation method. A study from Nepal has reported the same in $20 \%$, which is almost similar to the present study ${ }^{17}$. Culture positivity by conventional blood culture method was $13.3 \%$ in this study. A study from Chandigarh has reported overall blood culture positivity of $9.94 \%{ }^{18}$ which is less than this study. Culture positivity of enteric fever by the lytic centrifugation method was $17.3 \%$ in this study. Different studies from USA have shown a better recovery of bacteria by the lytic centrifugation method, in comparison to the conventional blood culture method ${ }^{11,13-14,19}$. However, the lytic blood culture method yielded greater culture positivity $(17.3 \%)$, than the conventional method in our study. Almost similar result was found by Indian studies showed a higher positivity by lytic centrifugation method as compared to conventional method ${ }^{20-21}$. Another study from Bangladesh by Begum et $\mathrm{al}^{22}$ showed that out of 100 clinically diagnosed typhoid fever, 14 were blood culture positive for $S$. typhi by conventional method. In this study, 6 (4\%) of the patients with blood culture-positive by lytic centrifugation method were negative by conventional method. This may be due to such patient taking antibiotic. The lytic centrifugation system effectively recovers $S$. typhi and $S$. paratyphi from blood specimen by neutralizing some antibiotic ${ }^{15}$.

Present study, Total contamination by lytic centrifugation method was low $(0.5 \%)$, as compared to the conventional blood culture method (5.0\%). Kelly et al ${ }^{19}$ have reported $3 \%$ contamination by the lytic centrifugation method. By the conventional method, contamination as reported by Henry et $\mathrm{al}^{14}$ was slightly higher (3.2\%) than the lytic centrifugation method (3\%). However, few studies have reported greater contamination rates with the lytic centrifugation method (4.4-9.6\%), than conventional method $(0.6-2.7 \%)^{11,14,19}$. According to the standards published by the American Society for Microbiology, the rate of blood culture contamination should not exceed $3.0 \%{ }^{23}$. Therefore, in this study, the contamination rate by the conventional method is not acceptable. A current study from the United Kingdom has also reported $7.4 \%$ contamination from blood cultures ${ }^{24}$. Therefore, this highlights the need for regular training and education of health care professionals, who collect blood samples and laboratory technicians who process the samples.

The relative low sensitivity of the blood culture in diagnosing enteric fever is a common phenomenon. It may be due to indiscriminate use of antibiotics and difficulties in obtaining adequate volume of blood for culture from children ${ }^{25-26}$. Blood culture has the promise of diagnosis in the first week and is very specific, but its sensitivity is poor due to various factors. Sensitivity of cultures can be affected by antibiotic treatment, type of culture medium, length of incubation and variations of bacteraemia $^{27-28}$.

In present study, majority of the blood culture negative cases gave history of taking different antibiotics for variable periods. Antibiotic treatment prior to collection of sample inhibits the growth on blood cultures ${ }^{16,29}$. Similar finding were found by Lin and others, where they showed that the S. typhi was recovered from $5.3 \%$ of patients with prior antibiotic intake versus $5.8 \%$ without prior antibiotics $^{30}$.

The rapid confirmation of the etiological agent would facilitate an early institution of appropriate antimicrobial therapy, thereby reducing clinical morbidity especially in an endemic population. It is worth while practicing lytic centrifugation method of blood culture for the accurate diagnosis of enteric fever in developing countries.

\section{Conclusion}

It is concluded that the lytic blood culture method was much better than conventional method yielding very good result within a short time. In resource restricted developing country like Bangladesh, where advanced technologies like automated culture systems (BACTEC, FAN) and PCR are not 
feasible because of those technologies require extensive infrastructure, specialized skilled personnel. On other hand, lytic method is cheap, easy, and simple; do not require any specialized equipments and uses chemicals and media which are readily available. So, lytic blood culture is a suitable method for rapid identification of aetiological agents, it would permit the clinician to institute appropriate therapy, thereby reducing hospital stay, morbidity and mortality in patients with enteric fever.

\section{References}

1. Jenkins C, Gillespie SH. Salmonella Infections. In: Gordon C, Cook, Zumla AI, eds. Manson's Tropical Diseases, 22nd edition. China: Saunders Elsevier 2009: 931-952

2. Singh B. Typhoid fever: epidemiology. J Indian Academy Clin Med 2001; 2(1): 17-20

3. Anggraini R, Handoyo I, and Aryati. DOT- EIA typhoid test using Omp salmonella typhi local phage type antigen to support the diagnosis of typhoid fever. Folia Medica Indonesiana 2004; 40(1):10-20

4. Jerrold $\mathrm{R}$ and Turner. The gastrointestinal tract: Salmonellosis, In : Kumar V, Abbas AK, Fausto and Aster JC, editors, Robins and Cotran Pathologic Basis of Disease, $8^{\text {th }}$ edition India, Elsevier, 2010; 801

5. Crump JA, Luby SP, Mintz ED. The global burden of typhoid fever. Bull World Health Organ 2004; 82: 346353

6. Brooks AW, Hossain A, Goswami D, Sharmin AT, Nahar $\mathrm{K}$, Alam K, et al. Bacteraemic Typhoid fever in children in an urban slum, Bangladesh. Emerg Infect Dis 2005;11(2): 326-329

7. Zwadyk, P Enterobacteriaceae: Salmonella and Shigella, Intestinal pathogens, in Joklik, WK, Willet, HP, Amos, B and Wilfert, CM editors, Zinsser Microbiology, $20^{\text {th }}$ editon, USA, Appleton and Lange 1992: 556-565

8. Gasem MH, Dolmans WM, Isbandrio BB, Wahyono H, Keuter M, Djokomoeljanto, R et al. Culture of Salmonella typhi and Salmonella paratyphi from blood and bone marrow in suspected typhoid fever. Trop Geograph Med 1995;47(4):164-167

9. Wain J, Bay PV, Vinh H, Duong NM, Diep TS, Walse AL et al. Quantitation of bacteria in bone marrow from patients with typhoid fever: relationship between counts and clinical features. J Clin Microbiol 2001;39(4):15711576

10. Betly A, Daniel FF, Alice SS. Blood stream infection, In: Baily and Scott's Diagnostics Microbiology, Mostby, Missouri, USA, $12^{\text {th }}$ edition, 2010; 865-880

11. Henry NK, McLimans CA, Wright AJ, Thompson RL, Wilson WR, Washington JA., 2nd Microbiological and clinical evaluation of the isolator lysis-centrifugation blood culture tube. J Clin Microbiol 1983;17:864-9

12. Dorn GL, Land GA, Wilson GE. Improved blood culture technique basedon centrifugation. clinical evaluation. J Clin Microbiol 1979; 9:391-396

13. Kiehn TE, Wong B, Edwards FF, Armstrong D. Comparative recovery of bacteria and yeasts from lysiscentrifugation and a conventional blood culture system. J Clin Microbiol 1983;18:300-4
14. Henry NK, Grewell CM, Van Grevenhof PE, Ilstrup DM, Washington JA., 2nd Comparison of lysis-centrifugation with a biphasic blood culture medium for the recovery of aerobic and facultatively anaerobic bacteria. J Clin Microbiol 1984;20:413-6

15. Rechard and Thompson. Specimen collection, transport and processing, In: Murray PP, Baron EJ, Jorgensen JH, Landry ML, Pfaller MA. Manual of Clinical Microbiology, 9th edition, Washington DC, 2007; 1: 310.

16. Chessbrough M. District Laboratory Practice in Tropical Countries. $2^{\text {nd }}$ ed. Vol.2. New York, USA: Cambridge University 2010; 184- 186

17. Cheesbrough M. District Laboratory Practice in Tropical Countries. $2^{\text {nd }}$ ed. Vol.2. Cambridge: Cambridge University Press; 2006; 127

18. Shrestha P, Das BK, Bhatta NK, Jha DK, Das B, Setia A, et al. Clinical and Bacteriological Profiles of Blood Culture Positive Sepsis in Newborns. J Nepal Paediatr Soc 2007;27:64-7

19. Kelly MT, Buck GE, Fojtasek MF. Evaluation of a lysiscentrifugation and biphasic bottle blood culture system during routine use. J Clin Microbiol 1983;18:554-7

20. Mantur BG, Mangalgi SS. Evaluation of conventional castaneda and lysis centrifugation blood culture techniques for diagnosis of human brucellosis. J Clin Microbiol 2004;42:4327-8

21. Sinha K, Tendolkar U, Mathur M. Comparison of Conventional Broth Blood Culture Technique and Manual Lysis Centrifugation Technique for Detection of Fungemia. Indian J Med Microbiol 2009;27:79-80

22. Begum Z, Hossain MA, Shamsuzzaman AK, Ahsan MM, Musa AK, Mahmud MC. et al. Evaluation of Typhidot (IgM) for Early Diagnosis of Typhoid Fever, Bangladesh. J Med Microbiol 2009; 03(1):10-13.

23. Ernst DJ. Controlling blood-culture contamination rates. (quiz 20-1).MLO Med Lab Obs 2004;36:14-8

24. Raja N, Parratt D, Meyers M. Blood culture contamination in a district general hospital in the UK: a 1-year study. Healthc Infect 2009;14:95-100

25. Bhutta ZA and Mansurali N. Rapid serologic diagnosis of paediatric typhoid fever in an endemic area: a prospective comparative evaluation of two dot-enzyme immunoassays and the Widal test. Am. J. Trop. Med. Hyg 1999; 61(4), 654-657.

26. Mohanty SK and Ramana KV. Single and Unpaired Sera Tube Widal Agglutination Test in Enteric Fever, The Saudi Journal of Gastroenterology 2007;13(4): 213

27. Miller IS and Pegues DA. Salmonella species, including Salmonella typhi, In: Mandell, Douglar and Bennetts principles and practice of infectious disease, Mandell GL, Bennett JE, Dolin R. $5^{\text {th }}$ edition, Philadelphia, Churchill Livingstone, 2000; pp. 2344-2263

28. Gilman R, Terminel M, Levine M, Mendoza PH and Hornick RB.The relative efficacy of blood, urine, rectal swab, and rose spot cultures for recovery of $S$. typhi in typhoid fever. Lancet 1975;1(7918):1211-1213

29. Gasem MH, Smits HL, Goris MG and Dolmans WM. Evaluation of a simple and rapid dipstick assay for the diagnosis of typhoid fever in Indonesia. J Med Microbiol. 2002;51(2):173-177

30. Lin FY, Ho VA, Bay PV et al. The Epidemiology of typhoid fever in the Dong Thap province, Mekong Delta region of Vietnam, Am J Trop Med Hyg. 2000;62(5):644648 